\title{
Monolayer Stress Microscopy: Limitations, Artifacts, and Accuracy of Recovered Intercellular Stresses
}

\author{
Dhananjay T. Tambe ${ }^{1 *}$, Ugo Croutelle ${ }^{2}$, Xavier Trepat ${ }^{3,4}$, Chan Young Park ${ }^{1}$, Jae Hun Kim ${ }^{1}$, Emil Millet ${ }^{1}$, \\ James P. Butler ${ }^{1,5}$, Jeffrey J. Fredberg ${ }^{1}$
}

1 Department of Environmental Health, Harvard School of Public Health, Boston, Massachusetts, United States of America, 2 Department of Physics, University Paris VII Diderot, Paris, France, $\mathbf{3}$ Institute for Bioengineering of Catalonia - ICREA, Barcelona, Spain, 4 Unitat de Biofísica i Bioenginyeria - CIBERES, Universitat de Barcelona, Barcelona, Spain, $\mathbf{5}$ Department of Medicine, Harvard Medical School, Boston, Massachusetts, United States of America

\begin{abstract}
In wound healing, tissue growth, and certain cancers, the epithelial or the endothelial monolayer sheet expands. Within the expanding monolayer sheet, migration of the individual cell is strongly guided by physical forces imposed by adjacent cells. This process is called plithotaxis and was discovered using Monolayer Stress Microscopy (MSM). MSM rests upon certain simplifying assumptions, however, concerning boundary conditions, cell material properties and system dimensionality. To assess the validity of these assumptions and to quantify associated errors, here we report new analytical, numerical, and experimental investigations. For several commonly used experimental monolayer systems, the simplifying assumptions used previously lead to errors that are shown to be quite small. Out-of-plane components of displacement and traction fields can be safely neglected, and characteristic features of intercellular stresses that underlie plithotaxis remain largely unaffected. Taken together, these findings validate Monolayer Stress Microscopy within broad but well-defined limits of applicability.
\end{abstract}

Citation: Tambe DT, Croutelle U, Trepat X, Park CY, Kim JH, et al. (2013) Monolayer Stress Microscopy: Limitations, Artifacts, and Accuracy of Recovered Intercellular Stresses. PLoS ONE 8(2): e55172. doi:10.1371/journal.pone.0055172

Editor: Sanjay Kumar, University of California, Berkeley, United States of America

Received August 5, 2012; Accepted December 19, 2012; Published February 28, 2013

Copyright: (C) 2013 Tambe et al. This is an open-access article distributed under the terms of the Creative Commons Attribution License, which permits unrestricted use, distribution, and reproduction in any medium, provided the original author and source are credited.

Funding: This research was supported by the Spanish Ministry for Science and Innovation (BFU2009-07595 and BFU2012-38146), the European Research Council (Grant Agreement 242993) and the National Institutes of Health (R01HL102373, R01HL107561). The funders had no role in study design, data collection and analysis, decision to publish, or preparation of the manuscript.

Competing Interests: The authors have declared that no competing interests exist.

* E-mail: dhananjay@alumni.brown.edu

\section{Introduction}

The human body is a cooperative of about 80 trillion cells, each one of which receives constantly from its neighbors both chemical and physical signals [1]. The individual cell then integrates these signals and responds through apoptosis, proliferation, differentiation, or migration. For example, pivotal events in the biology of the stem cell, the differentiated cell, and the cancer cell are increasingly understood to be of a mechanical nature [2-15]. These basic processes, in turn, underlie collective cellular processes that include morphogenesis, injury and repair, growth, regeneration, and cancer.

In collective migratory processes, cells ordinarily move not as individual entities but as collective sheets, ducts, strands, or clusters [8]. It is well established that each individual cell can follow preset chemical, adhesive, or mechanical gradients (chemotaxis, haptotaxis, and durotaxis, respectively [16-18]), but how each cell can coordinate its migration with that of immediate neighbors has defied full comprehension. For the cell-cell pair that is studied in isolation, mechanical stress exerted across the cell-cell junction has been recently quantified and is conceptually straightforward $[19,20]$. For the integrated multicellular monolayer sheet, by contrast, mechanical stresses exerted across the multiple cell-cell junctions amongst numerous immediate neighbors have been more difficult to quantify experimentally.

For the particular case of the cellular monolayer in vitro, the stresses exerted between a cell and its neighbors can now be mapped using the method called Monolayer Stress Microscopy (MSM) [21,22]. Using MSM, Tambe et al. [22] have recently shown that the stresses within and between cells comprising an advancing monolayer sheet define a remarkably rugged stress landscape. And within that landscape, the individual cell tends to migrate along the local orientation of maximal principal stress in a collective migratory process referred to as plithotaxis [21-25]. Collective cellular migration, a heterogeneous stress landscape, and plithotaxis all arise in the context of multicellular cooperative systems but logically cannot arise in single cells or in isolated cellcell pair interactions.

Implementation of MSM begins with recovery of local tractions exerted by the monolayer upon its substrate. As originally described [26,27], the traction recovery procedure is twodimensional in the sense that components of both the local traction field and the local displacement field that are out of the plane of the monolayer are assumed to be negligible. Others have subsequently disputed the validity of this assumption on the grounds that out-of-plane tractions and out-of-plane displacements may be appreciable $[28,29]$. For estimation of in-plane tractions, they have argued that three dimensional methods are necessary because out-of-plane events invalidate two-dimensional methods $[28,29]$. We disagree with this argument, and present in Results and Discussion detailed supporting evidence.

Whether or not two-dimensional conditions might obtain, still other assumptions are required. The distribution of internal 
stresses within the monolayer is computed from the recovered inplane tractions based on the two-dimensional balance of forces in the cell plane as demanded by Newton's laws [22]. As described below, this balance of forces is expressed as a two-dimensional elasto-static boundary value problem (BVP) with a source term corresponding to the local substrate traction. To solve this problem requires two key assumptions. The first involves the physical conditions defined by stresses, displacements, or some combination thereof at the monolayer boundary. The optical fieldof-view is sometimes limited to a small region of the monolayer, and the boundary conditions at this limit are unknown. The second involves the elastic properties of the monolayer itself. The monolayer elastic properties are unknown as well. Concerning these unknowns, Tambe et al. [22] made certain assumptions and performed limited analysis supporting their plausibility.

Here we reexamine these assumptions quantitatively in detail. We begin by formulating the problem in two dimensions and highlighting the necessary assumptions. We go on to consider boundary conditions and associated ambiguities, and then describe our analytical, numerical, and experimental approaches. Taken together, these approaches show quantitatively that errors attributable to out-of-plane tractions and displacements, and to cell material properties, are slight, and that errors attributable to unknown boundary conditions, if suitably handled, are manageable.

\section{Governing equations}

We consider a monolayer comprising a collection of contiguous cells which forms a sheet that is flat and thin (Fig. la). By flat and thin we mean that, compared with the lateral span $(L)$ of the monolayer, its radius of out-of-plane curvature is large and its height $(h)$ is small. In that case, stresses within the monolayer $(\sigma)$ and underlying tractions exerted by the monolayer upon its substrate $(T)$ are taken to be planar with no out-of-plane contributions $\left(\sigma_{z z}=\sigma_{z x}=\sigma_{z y}=T_{z}=0\right.$, Fig. 1b); the contribution of out-of-plane tractions, and the accuracy of in-plane traction recovery is assessed in Results and Discussion.

(a)

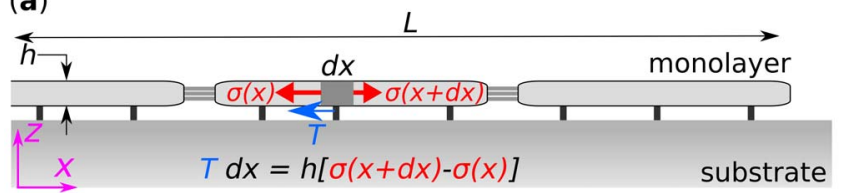

(b)
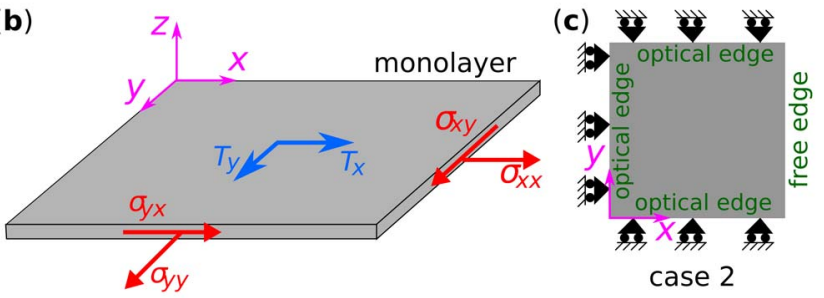

Figure 1. Balance of forces considered in MSM. (a) Cell monolayer is considered as a thin sheet of cells. Each cell in the monolayer exerts traction, T, on the substrate. According to the Newton's second law, the tractions are balanced by local monolayer stress, $\sigma$, such that, in the one dimensional force balance, $T d x=h[\sigma(x+d x)-\sigma(x)]$. (b) The force balance is ensured only in the xy plane. Variation of stresses across the thickness is assumed to be negligible. (c) In classical wound healing assay, also referred to as case 2, the optical field-of-view has three optical edges and a free edge. For boundary conditions, all edges have shear stress to be zero. In addition, the free edge has normal stress to be zero and the optical edge has normal displacement to be zero. doi:10.1371/journal.pone.0055172.g001
If inertial effects are negligible, then according to Newton's second law, the local traction must be balanced by local gradients in monolayer stresses. This is simply a force balance argument, which implies

$$
\frac{\partial \sigma_{x x}}{\partial x}+\frac{\partial \sigma_{x y}}{\partial y}=T_{x}^{*}, \frac{\partial \sigma_{x y}}{\partial x}+\frac{\partial \sigma_{y y}}{\partial y}=T_{y}{ }^{*},
$$

where $T^{*}=-T / h$. If the monolayer is homogeneous and isotropic, then there are only two independent elastic constants, which can be described by the Young's modulus $E$ and the Poisson's ratio $v$; we consider the viscous properties in Discussion. In this case, the relationship between strain and stress is given by

$$
\left[\begin{array}{c}
\varepsilon_{x x} \\
\varepsilon_{y y} \\
\varepsilon_{x y}
\end{array}\right]=\frac{1}{E}\left[\begin{array}{ccc}
1 & -v & 0 \\
-v & 1 & 0 \\
0 & 0 & 1+v
\end{array}\right]\left[\begin{array}{c}
\sigma_{x x} \\
\sigma_{y y} \\
\sigma_{x y}
\end{array}\right]
$$

To ensure that displacements can be integrated from strains yielding a single valued vector field, Saint-Venant's compatibility relation for strains must be satisfied, $\frac{\partial^{2} \varepsilon_{x x}}{\partial y^{2}}+\frac{\partial^{2} \varepsilon y y}{\partial x^{2}}=2 \frac{\partial^{2} \varepsilon x y}{\partial x \partial y}$. Together with Eqs. 1 and 2, we then obtain the Beltrami-Michell compatibility equation,

$$
\left(\frac{\partial^{2}}{\partial x^{2}}+\frac{\partial^{2}}{\partial y^{2}}\right)\left(\sigma_{x x}+\sigma_{y y}\right)=(1+v)\left(\frac{\partial T_{x}^{*}}{\partial x}+\frac{\partial T_{y}^{*}}{\partial y}\right) .
$$

Eqs. 1 and 3 are sufficient to ensure local force balance; the source terms arising from tractions and the boundary conditions then determine stresses everywhere within the monolayer.

Since the monolayer always remains in mechanical equilibrium, Newton's laws demand that this force balance must be independent of material properties of the monolayer. The recovered stresses, however, are not independent of the specific material properties. Tambe et al. [22] had asserted incorrectly that the specific material properties of the monolayer have no effect on the recovered distribution of intercellular stresses; while this statement is indeed true for one-dimensional systems, it need not be true in two-dimensional systems largely due to effects of Poisson's ratio. Eqs. 1 and 3 show that if the material properties are homogeneous, then the distribution of internal stresses does not depend upon $E$ but does depend upon $v$. However, we show below that variations in $v$ in two dimensions can have at most only a small effect. Thus, the prior assertion of Tambe et al. [22] that recovered stresses are independent of specific material properties remains true in one dimension and is approximately true in two dimensions.

In MSM, stresses are determined with standard finite element procedures which use Eqs. 1 and 2. In this case, Eq. 3 is satisfied automatically. Below we follow the standard approach and to do so we assign values for $v$ and $E$. In Results we assess the influence of $v$ on the recovered monolayer stresses. In contrast, the assigned value for $E$ is entirely arbitrary, and does not influence the recovered monolayer stress distribution.

If the monolayer is neither isotropic, homogeneous, nor elastic, then the Beltrami-Michell compatibility equation becomes more complex, In Results and Discussion we deal with these cases as well.

\section{Boundary conditions}

In most experimental monolayer systems, two types of boundaries require consideration (Fig. 1c). The first is the edge 
of the advancing monolayer front that is bounded by a cell-free, traction-free region; we call this the free edge. The second is the edge of the optical field-of-view that is bounded on one side by cells that are outside the optical field-of-view, and on the other side by cells that are inside the optical field-of-view; we call this the optical edge. Boundary conditions at the free edge are known (monolayer boundary stresses are zero), but boundary conditions at the optical edge are unknown.

To overcome the difficulty posed by the boundary conditions at the optical edge, Tambe et al. [22] assumed that along the optical edges $u_{i} n_{i}=0, \sigma_{i j} n_{j} t_{i}=0$, (summation convention used) where $u$ is the displacement, $n$ and $t$ are the normal and tangent unit vectors at the optical edge; we call this a symmetric boundary condition. These boundary conditions imply that the region of interest (Fig. 1c) is a repeatable unit of the monolayer; the free edge defines the boundary of the monolayer, but the optical edge defines the interior edge across which the unit repeats as a mirror image. As such, Fig. 1c represents a monolayer that is infinite in extent along the $y$-direction and twice the size of the region of interest along the $x$-direction.

Recognizing that this assumption is ad hoc and might engender errors, Tambe et al. [22] showed by experimental means that these errors are largest in regions nearest the optical edge and decay rapidly with distance from the optical edge, becoming negligible beyond a distance of $20 \%$ of the length of the boundary. In this connection, the subsequent work of Hur et al. [28] assumes optical edges to be stress free (both normal and tangential stresses are zero); in Discussion, we provide below a quantitative comparison of the approach of Hur et al. [28] with that of Tambe et al. [22].

At the optical edge, Tambe et al. [22] constrained the normal displacements to be zero (Fig. 1c). In this case, a nonzero stress along the $x$-direction will induce stress along the $y$-direction which is partly artifactual. Although this induced stress along the $y$ direction is associated with the boundary conditions, its magnitude depends upon $v$. Therefore, in the approach of Tambe et al. [22], boundary artifacts and material artifacts are coupled.

In the measurement of monolayers bounded by free edges alone (Fig. S2c in File S1), there are no optical edges, and as such, there are no unknown boundary conditions and no associated boundary artifacts. We take advantage of this fact below.

\section{Analysis}

Based on geometric scaling arguments, we first estimate the magnitude of out-of-plane tractions in comparison with in-plane tractions; based on analytic arguments, we then quantify the errors in recovered in-plane tractions when out-of-plane displacements are neglected. This is followed by an analysis of the errors in monolayer stresses attributable to the assumed simplified material properties of the cells. We conclude by considering the distinction between monolayers whose entire extent falls within the microscopic field-of-view versus those that extend outside the field-ofview.

\section{Analytic assessments}

Out-of-plane tractions and displacements. Concerning traction recovery, we first consider the role of out-of-plane tractions, $T_{z}$ [27]. Based purely on geometric arguments, we begin with a preliminary estimation of the error. For a semiinfinite elastic medium, we then derive the full analytic Fourier representation of substrate tractions and displacements in three dimensions.

\section{Experimental assessments}

To assess the accuracy of recovered monolayer stresses experimentally, we start by considering the monolayer bounded by free edges alone; we call such a monolayer a cell island (Fig. S2c, for experimental protocol see Supporting Information S1, in File S1). Stresses computed over the entire cell island have two useful properties. As noted above, the first property is the absence of optical edges and resulting boundary artifacts; accordingly we take these recovered stresses as being a gold standard. By comparison, we can then assess boundary artifacts in subsystems that include optical edges. The second property is that the absence of boundary artifacts makes these stresses ideal to assess MSM's artifacts that might be attributable to the assumptions of material incompressibility and homogeneity.

In this analysis, we compute local principal stress components $\left(\sigma_{\max }\right.$ and $\left.\sigma_{\min }\right)$, and focus on the local average normal stress $\left(\sigma_{\max }+\sigma_{\min }\right) / 2$ (which is the average local tension), the local maximum principal orientation (which is the axis of $\sigma_{\max }$ or local highest tension), and the local maximum shear stress $\left(\sigma_{\max }-\sigma_{\min }\right) / 2$ (which is a measure of the local stress anisotropy).

Effects of material properties of the monolayer. For $v$, reported values range from 0.3 to 0.5 [30]. To assess the influence of choosing $v$ on recovered monolayer stresses, we recomputed the stress field using $v=0.3$ and then compared the two stress fields.

To assess the effect of heterogeneity of $E$, we first considered the monolayer with homogeneous elastic properties where $v=0.5$ and computed the local average normal stresses. Recognizing that the cell stiffness is closely related to the cytoskeletal stress [31-33], we amplified this effect by taking the map of average normal stress to represent the non-uniform distribution of $E$, and then recalculated the stress field. We then compared the stress field recovered using homogeneous $E$ with the stress field recovered using heterogeneous $E$.

Effects of boundary conditions. To quantify boundary artifacts, as in the approach used by Tambe et al. [22], we computed stresses using two different methods. In the first method, stresses were computed by solving the equations of equilibrium over the entire island; we call this case 1 (the gold standard). In the second method, stresses were computed by following the approach used by Tambe et al. [22], i.e., solving the equations of equilibrium only in the region of interest that is bounded by optical edges on three sides; we call this case 2 (Fig. 1c). The effect of the assumed boundary conditions was quantified by comparing the recovered stresses recovered from case 2 with those from the artifact free stresses computed from case 1 .

\section{Numerical assessments}

Propagation of the boundary artifacts. To analyze further how boundary perturbations and associated artifacts propagate into the region of interest, we imposed sinusoidal perturbations at optical edges. For example, along the right optical edge in Fig. 1c, we replaced the condition of zero normal displacements, $u_{i} n_{i}=u_{n}=0$, with the condition of sinusoidal fluctuations, $u_{n}(y)=\hat{u}_{n} \sin (2 \pi y / \lambda)$, where $\hat{u}_{n}$ is the amplitude, $\lambda$ is the wavelength of the perturbation, and $y$ is the distance along the optical edge, with all other boundaries unchanged. We did so for wave lengths $\lambda=L / 2, L / 8$. A similar set of calculations were performed with shear stress perturbations at the optical edge, $\sigma_{x y}(y)=\hat{\sigma}_{x y} \sin (2 \pi y / \lambda)$, where $\hat{\sigma}_{x y}$ is the amplitude of the perturbation. Each of the perturbations, induced stresses in the monolayer. Spatial variation of these induced stresses is then plotted as a function of distance from the optical edge (Supporting Information S6 in File S1). 
Two other monolayer geometries are of interest. The first is the monolayer bounded by two optical edges separated by two free edges (Fig. S2a in File S1). The second is the monolayer bounded by optical edges on all four sides (Fig. S2b in File S1). These cases are treated in Supporting Information S4 in File S1.

\section{Results}

\section{Analytic Results}

Scaling analysis of out-of-plane tractions and their effects. There are at least three important length scales in the problem. (1) The lateral extent of in-plane traction fluctuations $l$, quantified by the inverse of a typical wavenumber $k$ in Fourier space or an autocorrelation length in real space; (2) substrate thickness $H$; and (3) monolayer height $h$. The importance of the relationship of substrate thickness to lateral extent of traction fluctuations $(k H$ or $H / l)$ has been dealt with before [34,35].

The remaining dimensionless number is $k h$, or, equivalently $h / l$. It is clear that if the monolayer height is large compared to length scale for traction fluctuations, then nothing in the $z$ direction can be neglected. This might be the case for an isolated cell that is cuboidal or rounded up with stress fibers draped over a big nucleus. In this regime, errors associated with measuring inplane displacements alone cannot be neglected, and are not treated here.

In the case of extended monolayers, by contrast, geometry alone gives bounds on the errors. Suppose there are true traction vectors of unit magnitude acting at two remote points separated by $l>>h$; their angle $\vartheta$ with respect to the $x, y$ plane is at most $O(h / l)$. Since the recovered $T_{x}$ and $T_{y}$ carry a factor of $\cos \vartheta=1-O\left(\vartheta^{2}\right)$, it follows that they differ from their true values only at second order in the ratio $h / l . T_{z}$ carries a $\sin \vartheta=O(\vartheta)$ dependence, implying an error that is first order in $h / l$.

If, for example, a typical lateral scale is $100 \mu \mathrm{m}$ and the monolayer cell height is $10 \mu \mathrm{m}$, then the geometric arguments above imply errors on the order of $1 \%$ for in-plane tractions and $10 \%$ for out-of-plane tractions. The ratio of out-of-plane to inplane traction would then be of order 0.1 , consonant with the findings of Hur et al. [28], who reported 0.37.

Exact analysis of out-of-plane displacements and their effects. If $v \neq 0.5$, then neglecting the non-zero effects of the $z$ component of displacement, $u_{z}$, introduces errors in $T_{x}$ and $T_{y}$. To quantify these errors we have extended the restricted two dimensional work of Butler et al. [36] to three dimensional space. A traction point source of unit force at the origin induces displacements at a point $(x, y)$ on the $z=0$ surface of a semiinfinite medium, given by the classical Boussinesq solution $\vec{u}=K \vec{T}$, where

$$
K(x, y)=\frac{A}{r^{3}}\left[\begin{array}{ccc}
(1-v) r^{2}+v x^{2} & v x y & (1-2 v) r x / 2 \\
v x y & (1-v) r^{2}+v y^{2} & (1-2 v) r y / 2 \\
-(1-2 v) r x / 2 & -(1-2 v) r y / 2 & (1-v) r^{2}
\end{array}\right],
$$

$\vec{T}=\vec{e} \delta^{2}(\vec{r})$ is the point source of traction, $\vec{e}$ is a (3 dimensional) unit vector, and $\delta(\cdot)$ is the Dirac delta function. The prefactor is given by $A=(1+v) / \pi E$, where $E$ and $v$ are Young's modulus and Poisson's ratio of the substrate. It is important to note that the classical Boussinesq solution is valid only when gradients of the displacements $\left(u_{x}, u_{y}, u_{z}\right)$ are small.

In Supporting Information S2 (in File S1), we show that in twodimensional Fourier space, this becomes

$$
\tilde{K}(\vec{k})=\frac{2 \pi A}{k^{3}}\left[\begin{array}{ccc}
(1-v) k^{2}+v k_{y}^{2} & -v k_{x} k_{y} & i(1-2 v) k_{x} k / 2 \\
-v k_{x} k_{y} & (1-v) k^{2}+v k_{x}^{2} & i(1-2 v) k_{y} k / 2 \\
-i(1-2 v) k_{x} k / 2 & -i(1-2 v) k_{y} k / 2 & (1-v) k^{2}
\end{array}\right] .
$$

We use this formulation to quantify the errors in the recovered tractions attributable to neglecting out-of-plane displacements. As there is no intrinsic or independent length scale or preferred direction, it suffices to examine in detail the behavior for, say, $k_{x}=1, k_{y}=0, k=1$, in which case we have

$$
\tilde{K}(\vec{k})=2 \pi A\left[\begin{array}{ccc}
(1-v) & 0 & i(1-2 v) / 2 \\
0 & 1 & 0 \\
-i(1-2 v) / 2 & 0 & (1-v)
\end{array}\right] .
$$

The recovery of tractions is then given by $\tilde{T}=\tilde{K}^{-1} \tilde{\vec{u}}$, where

$$
\tilde{K}^{-1}(\vec{k})=B\left[\begin{array}{ccc}
(1-v) & 0 & -i(1-2 v) / 2 \\
0 & 1 & 0 \\
+i(1-2 v) / 2 & 0 & (1-v)
\end{array}\right],
$$

and where $B=2(\pi A(3-4 v))^{-1}$.

We now consider a unit displacement that has threedimensional components $(\cos \vartheta, 0, \sin \vartheta)$, the first two being inplane and last out-of-plane. The tractions are given by,

$$
\left[\begin{array}{c}
\tilde{T}_{x} \\
\tilde{T}_{y} \\
\tilde{T}_{z}
\end{array}\right]=\tilde{K}^{-1}(\vec{k})\left[\begin{array}{c}
\cos \vartheta \\
0 \\
\sin \vartheta
\end{array}\right]=B\left[\begin{array}{c}
(1-v) \cos \vartheta-1 / 2 i(1-2 v) \sin \vartheta \\
0 \\
+1 / 2 i(1-2 v) \cos \vartheta+(1-v) \sin \vartheta
\end{array}\right] .
$$

The true in-plane traction is $T_{x}^{\text {true }}=B[(1-v) \cos \vartheta-1 / 2 i(1-2 v) \sin \vartheta]$. The recovered in-plane traction (neglecting the out-of-plane displacement) is $T_{x}^{r e c}=B(1-v) \cos \vartheta$. The departures from unity of the ratio $\left|T_{x}^{r e c}\right| /\left|T_{x}^{\text {true }}\right|$ quantifies the error in magnitude, while $\operatorname{Arg}\left[T_{x}^{r e c} / T_{x}^{\text {true }}\right]$ quantifies the error in phase. These quantities are plotted in Fig. 2.

When $v=0.5$, these errors vanish as expected. However, even when $v$ departs from 0.5 substantially, the error in recovered magnitude remains remarkably small (second order in $\vartheta$ ) and the error in phase also remains small (first order in $)$ ). This behavior parallels the geometric argument above.

For example, taking an extreme value for polyacrylamide gels [37], $v=0.4$, and taking $\vartheta=30^{\circ}$, the error in magnitude would be less than $1 \%$ and the error in phase would be approximately $5^{\circ}$.

Finally, we remark that for substrates of finite thickness, when the tractions are smoothly distributed over distances larger than gel thickness, the substrate deformation approximates pure uniform shear, and in this case Poisson's ratio in the substrate is irrelevant.

\section{Experimental Results}

Case 1: The isolated cell island. In an island of rat pulmonary microvascular endothelial (RPME) cells, tractions demonstrated extreme spatial fluctuations $\left(T_{x}\right.$, Fig. $3 \mathrm{~b}$; and $T_{y}$, Fig. 3c). These fluctuations are comparable to those previously reported $[22,27]$. Using these traction fields together with Eqs. 1 and 2, and assuming the monolayer elastic properties to be homogeneous and incompressible $(v=0.5)$, the resulting intercel- 

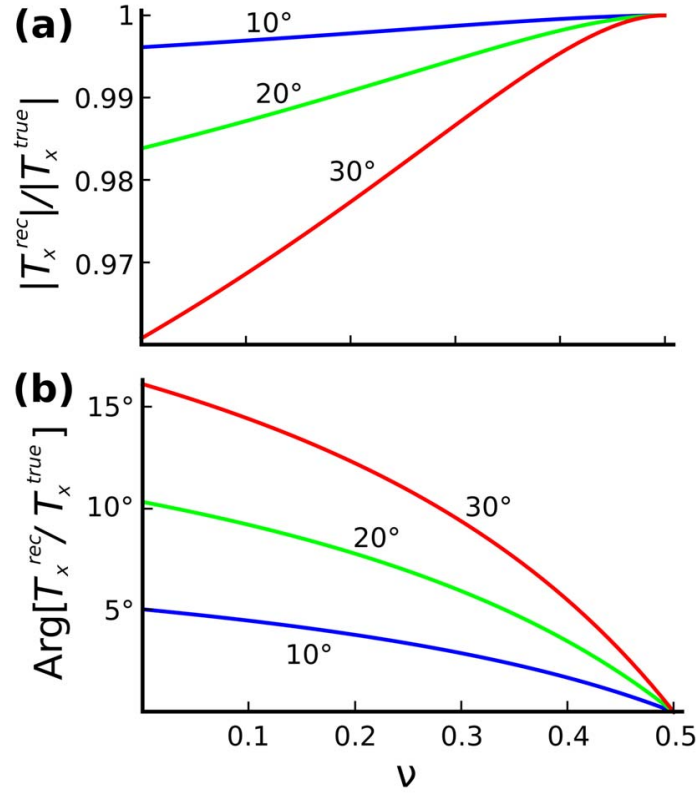

Figure 2. Accuracy of in-plane tractions as a function of Poisson's ratio when out-of-plane components of displacements are neglected. $\vartheta$ denotes the angle of the displacement vector relative to the $x, y$ plane. (a) Ratio of recovered in-plane traction to true in-plane traction. (b) Error in the phase of the recovered in-plane traction (degrees).

doi:10.1371/journal.pone.0055172.g002

lular stress landscape demonstrated the same characteristic ruggedness as observed previously by Tambe et al. [22] (Fig. 4a-c).

When we reduced the assumed value of $v$ from 0.5 to 0.3 , the resulting stress landscape changed relatively little (Fig. $4 d-\mathrm{d}$ ). Similarly, when we introduced dramatic heterogeneities in $E$ by assuming that $E$ is proportional to the average normal stress (Fig. 4b), again the changes were modest (Fig. 4g-i). To quantify these differences, we created scatter plots using stresses from every grid point. On the $E$-axis we plotted stresses computed for the monolayer where $v$ is 0.5 and $E$ is homogeneous, and on the $y$-axis we plotted stresses computed for the monolayer under the other two situations. Accordingly, Fig. $4 \mathrm{j}-\mathrm{l}$ represent sensitivity of stresses to the choice of $v$ (red points) and to the heterogeneity of $E$ (blue points).

When $v$ was reduced to 0.3 , the three stress measures (average normal stress, the maximum principal orientation, and the maximum shear stress) each tracked closely the line of identity and showed little variation (Fig. $4 \mathrm{k}-\mathrm{l}$, red points). Unlike changes associated with the magnitude of $v$, changes associated with the heterogeneity of $E$ were somewhat greater (Fig. $4 \mathrm{j}-1$, blue points). For average normal stress, the relationship was slightly sigmoidal (Fig. 4k, blue points); at low magnitudes the stresses were underestimated and at high magnitudes the stresses were overestimated. For maximum principal orientation, the points were symmetrically scattered around the line of identity (Fig. 4j, blue points). For maximum shear stress, the points were spread asymmetrically about the line of identity (Fig. 4l, blue points); at high magnitudes the stresses were overestimated.

Across all instances examined, the correlation coefficient $r^{2}$ was no smaller than 0.79 ; the slope $m$ was close to unity (0.92 to 1.1$)$; the intercept $c$ was $\left(-0.01^{\circ}\right.$ to $\left.0^{\circ}\right)$ for orientation of maximum principal orientation, and $(-0.23 \mathrm{~Pa}$ to $0.61 \mathrm{~Pa})$ for average normal stress and maximum shear stress.
Case 2: Subsystem bounded by optical edges on three sides. Compared to the gold standard (Fig. 5a-c), the stresses computed from case 2 were systematically different (Fig. 5d-f). The stresses away from the optical edges were closely similar, whereas the stresses close to the optical edges were appreciably different (Fig. 5g-i). Moreover, as proposed by Tambe et al. [22], the differences in the stresses were largely limited to a narrow band whose width is $20 \%$ of the length of the optical edge, depicted by the grey band in Fig. $5 \mathrm{a}-\mathrm{f}$.

When the entire stress map was compared, the agreement between the gold standard and stresses from case 2 was weak, as expected (Fig. 5j-l, blue points). But when the cropped maps (which excluded the boundary region depicted by the grey band) were compared, the agreement between stresses was far better (Fig. 5j-l, red points). Nonetheless, average normal stresses were slightly underestimated (Fig. 5k, red points), the maximum principal orientations were slightly biased along a diagonal of the monolayer geometry (Fig. 5j, red points), and the maximum shear stresses were rather weakly correlated (Fig. 5l, red points).

Across all instances examined, in the region away from the optical edge, the correlation coefficient $r^{2}$ was no smaller than 0.66 ; the slope $m$ was in the range 0.77 to 0.99 ; the intercept $c$ was $\left(-0.12^{\circ}\right.$ to $\left.-0.11^{\circ}\right)$ for orientation of maximum principal orientation, and $(-5.5 \mathrm{~Pa}$ to $-0.39 \mathrm{~Pa})$ for average normal stress and maximum shear stress.

\section{Numerical Results}

Propagation of the boundary artifacts. First, we considered an optical edge adjacent to the free edge, and imposed boundary perturbation comprising sinusoidal normal displacements $u_{n}(y)=\hat{u}_{n} \sin (2 \pi y / \lambda)$, where $\lambda=L / 2$ (Fig. 6a). These normal displacements induced average normal stress which decayed monotonically with distance from the boundary (Fig. 6b, and Fig. 6f, blue lines marked with circle); when the wavelength of the perturbation was smaller the decay was faster (Fig. 6d, and Fig. 6f, red lines marked with circle). Indeed, the decay length in each case was comparable to the wavelength of perturbation. While the decay of average normal stress was monotonic, the decay of maximum shear stress was not monotonic (Figs. 6c,e; and Fig. $6 f$ lines marked with cross). Finally, when the perturbed edge was the optical edge away from the free edge, the stresses were similar (Fig. 6h).

Next, we considered the boundary perturbations comprising sinusoidal shear stress $\sigma_{x y}(y)=\hat{\sigma}_{x y} \sin (2 \pi y / \lambda)$. Unlike the normal displacements perturbations, the shear stress perturbations induce stresses whose rate of decay depends on choice of the optical edge. When the perturbed edge was the optical edge away from the free edge, the induced stresses decayed faster (Fig. 6i, Supporting Information S5; Fig. S8; in File S1).

Analysis of case 3 and 4 produced results that were qualitatively similar to those of the analysis of case 2 (Supporting Information S4; Figs. S3-S7 and Fig. S14; in File S1).

\section{Discussion}

Our principal findings are these. For in-plane traction recovery and for in-plane monolayer stress recovery, the contributions of out-of-plane components of tractions and displacements are negligible. Second, optical edges cause artifacts that are mostly confined to regions near the optical edge but are otherwise homogeneous and small. Third, assumptions of homogeneity and incompressibility of the monolayer material cause artifacts that are also small. Finally, characteristic features of the recovered stress maps that underlie plithotaxis are insensitive to these artifacts, thus 

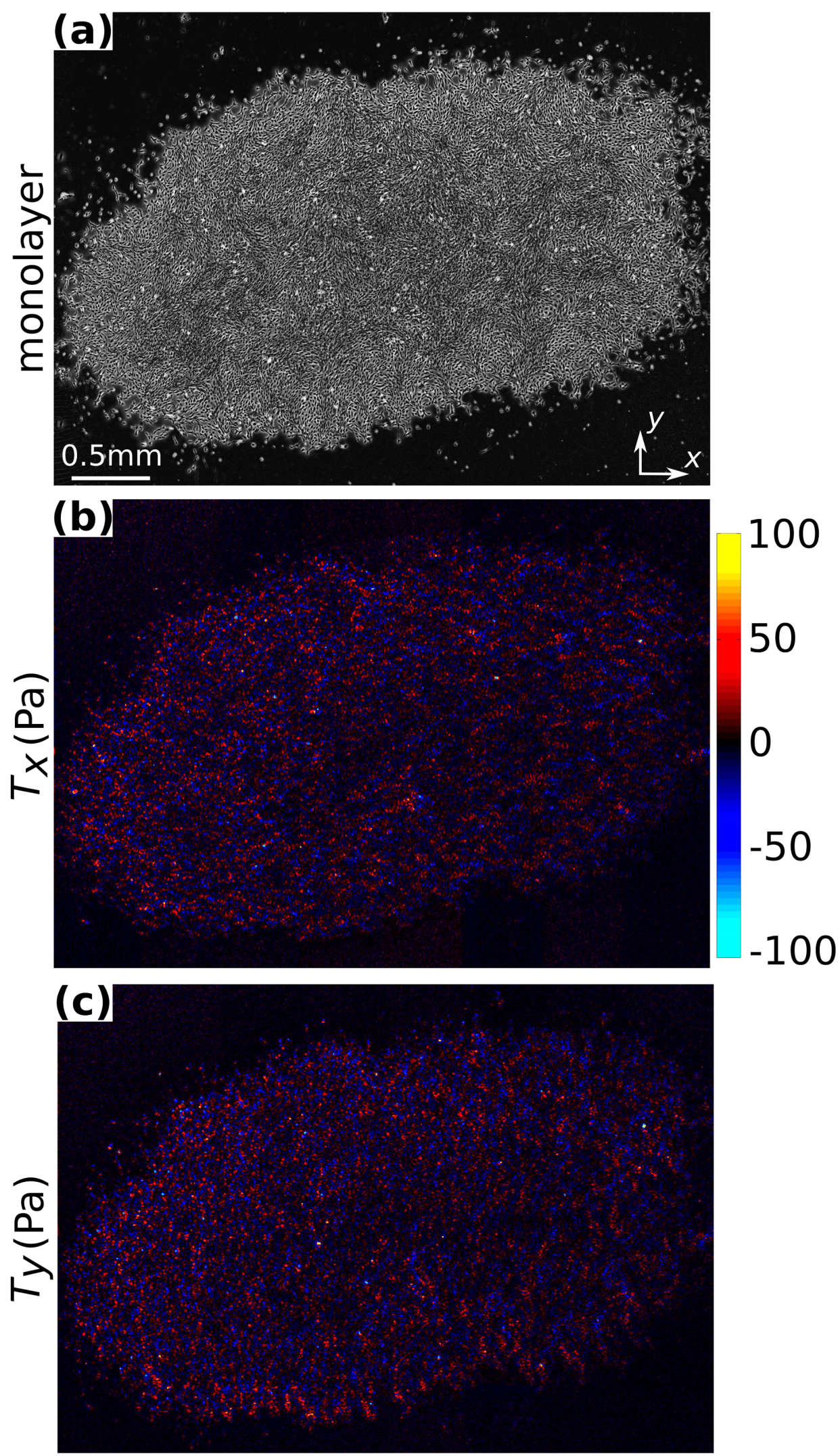

Figure 3. Substrate tractions for RPME cell island. (a) Island of RPME cells. (b,c) Two components of tractions, $T_{x}$ and $\mathrm{T}_{\mathrm{y}}$ respectively, applied by these cells on the substrate. Size of the cell island: $4.2 \mathrm{~mm} \times 2.6 \mathrm{~mm}$. doi:10.1371/journal.pone.0055172.g003 
max. principal orientation
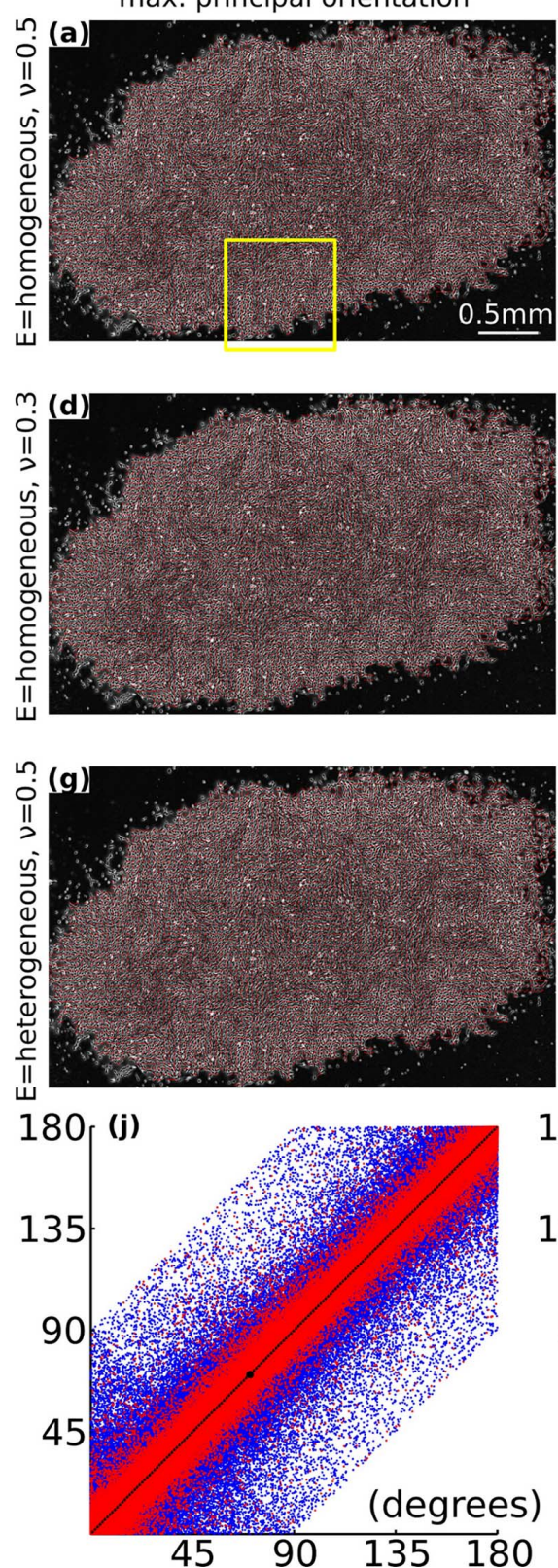

avg. normal stress $(\mathrm{Pa})$
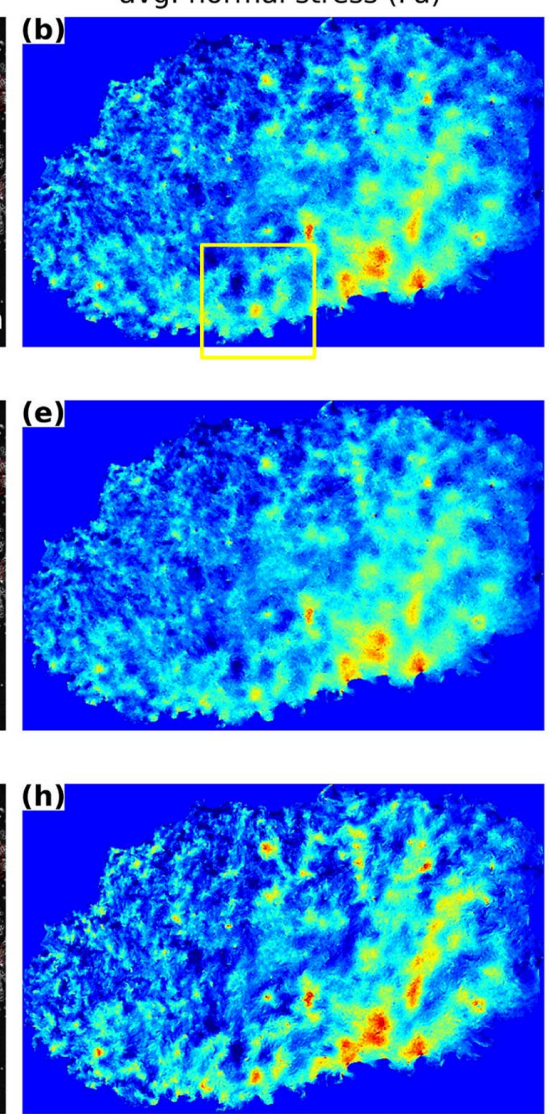
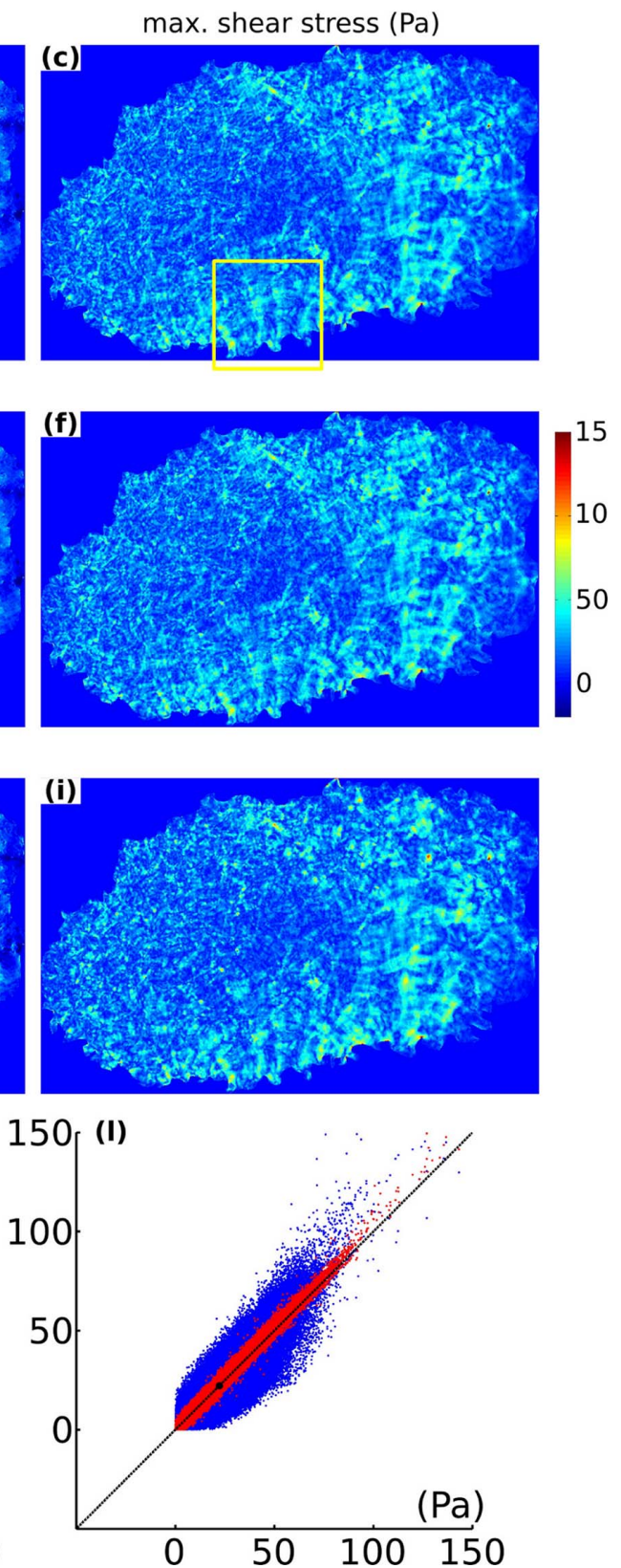

Figure 4. Sensitivity of the recovered monolayer stresses to change in the assumed elastic properties of the monolayer. (a) Map of maximum principal orientation (for enlarged version of this image, see Supporting Information S8; Figs. S11-13; in File S1), (b) map of average normal stress, and (c) map of maximum shear stress obtained by assuming monolayer elastic properties to be homogeneous with $v=0.5$. (d-f) The stress maps with $v=0.3$ instead. $(\mathrm{g}-\mathrm{i})$ The stress maps when $\mathrm{E}$ is heterogeneous with $v=0.5$, here $\mathrm{E}$ was assumed to be proportional to the map of the average normal stress (b). (j) Scatter plots for maximum principal orientation where, red points quantify effect of $v$ on the maps (a) and (d), and blue points quantify effect of heterogeneity of $\mathrm{E}$ on the (a) and (g). (k) Scatter plots for average normal stress, (I) scatter plots for maximum shear stress. Regression parameters for a straight line fit, $\mathrm{y}=\mathrm{mx}+\mathrm{c}$ in (j-l): blue points, (j) $m=0.96, c=-0.01^{\circ}, r^{2}=0.93$, (k) $m=1.1, c=-0.23 P a, r^{2}=0.91$, and (l) $m=0.97, c=-0.02 P a, r^{2}=0.79$; red points, (j) $m=1, c=0^{\circ}, r^{2}=0.99$, (k) $m=0.92, c=-0.03 P a, r^{2}=0.99$, and (l) $m=1.01, c=0.61 P a, r^{2}=0.99$. doi:10.1371/journal.pone.0055172.g004

demonstrating that the observation of plithotaxis is robust. Below we discuss in greater detail monolayer stress recovery, associated findings, and their implications.

\section{Measurement of out-of-plane tractions and} displacements is not necessary

Recent reports have asserted that three dimensional methods are necessary because the out-of-plane tractions or displacements can be sufficiently large to invalidate tractions and monolayer stresses recovered from two-dimensional methods [28,29]. To assess the validity of this assertion we address three key questions.

First, to what degree do out-of-plane displacements, which are unmeasured in MSM, impact recovery of in-plane tractions? When the substrate is incompressible $(v=0.5)$ and thick (Supporting Information S7; Fig. S10; in File S1), out-of-plane displacements and in-plane tractions become decoupled [35,38]. In that case errors attributable to ignoring out-of-plane events are 
max. principal orientation
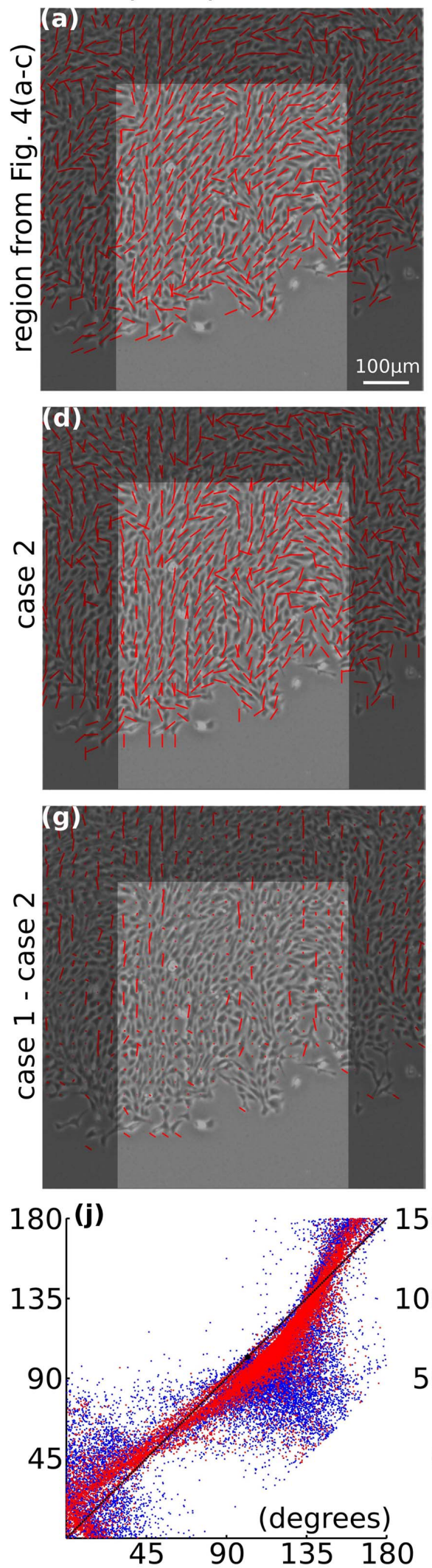

avg. normal stress $(\mathrm{Pa})$
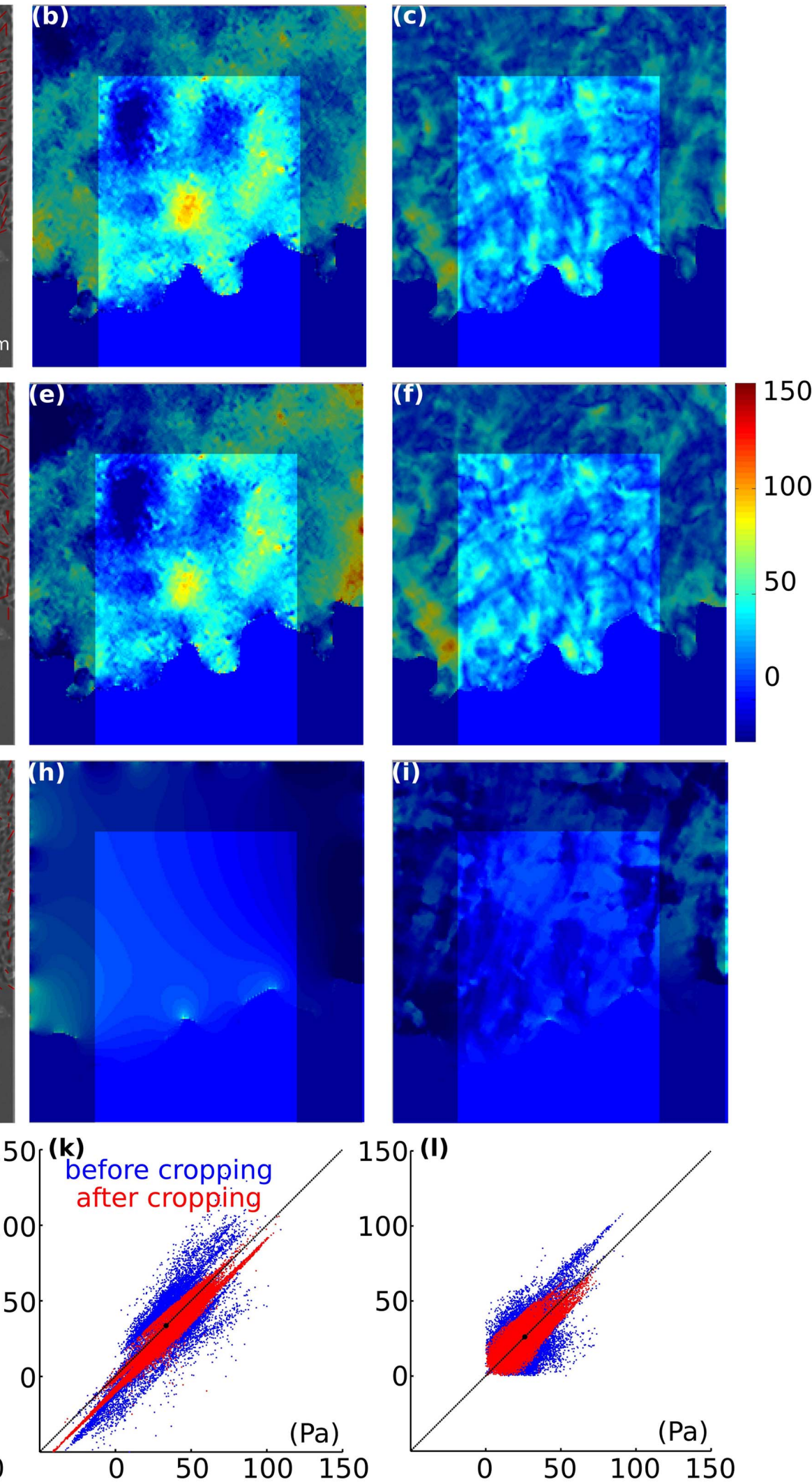

Figure 5. Influence of the optical edges on the monolayer stresses recovered for case 2. Maps of (a) maximum principal orientation, (b) average normal stress, and (c) maximum shear stress extracted from the region of interest (Fig. 4a-c, yellow rectangle). (d-f) Stress map obtained by limiting the solution of equilibrium equations to the region of interest. (g) Map of difference between (a) and (d). (h) Map of (b) minus (e). (i) Map of (c) minus (f). The grey 
band in (a-i) represents cropped region; width of this region was $20 \%$ of the length of the optical edge. (j) Scatter plots of maximum principal orientations for quantitative comparison between (a) and (d). For the blue points cropped region was included, for the red points cropped region was exclude. (k) Scatter plots for average normal stress, (I) scatter plots for maximum shear stress. Regression parameters for a straight line fit, $\mathrm{y}=\mathrm{mx}+\mathrm{c}$ in $(\mathrm{j}-\mathrm{l})$ : blue points, (j) $m=0.85, c=-0.11^{\circ}, r^{2}=0.88,(\mathrm{k}) m=1.01, c=-5.55 P a, r^{2}=0.93$, and (l) $m=0.75, c=-0.55 P a, r^{2}=0.61$; red points, (j) $m=0.77, c=-0.12^{\circ}, r^{2}=0.76,(\mathrm{k})$ $m=0.99, c=-2.58 P a, r^{2}=0.83$, and (I) $m=0.85, c=-0.39 P a, r^{2}=0.66$. Size of the region of interest is $830 \mu \mathrm{m} \times 830 \mu \mathrm{m}$.

doi:10.1371/journal.pone.0055172.g005

precisely zero. Accordingly, in that case both two-dimensional and three-dimensional traction algorithms will recover in-plane tractions that are identical. Remarkably, even when Poisson's ratio departs substantially from 0.5 and the out-of-plane displacements are extreme, analysis shows that the errors in both magnitude and phase of the recovered in-plane tractions remain negligible (Fig. 2).

Second, are the recovered in-plane tractions insensitive to the difference between cells adhering tightly versus loosely to one another, as asserted by [29] Notbohm et al. [29]? This assertion stands in contrast with previously reported experimental data obtained using the very same cell type (MCF10A) and demonstrating the sensitivity of MSM to distinguish between control and preparations where cell-cell junctions were disrupted with cadherin antibodies ([22] [Fig. 4]). We reason that the contrary conclusion of Notbohm et al. [29] follows from a very specific feature of their model. In particular, Notbohm et al. [29] modeled the interaction between cells and substrate by elastic springs normal to the cell-substrate plane. In such a model, to first order there is no shear stress (i.e. in-plane substrate tractions) applied by the cells on the substrate. By construction, therefore, only in those springs that depart from first order strain will there be any transmission of in-plane tractions, and this occurs preferentially at the island boundary.

Third, to what degree do nonzero out-of-plane displacements and out-of-plane tractions affect the recovered in-plane monolayer stresses? If out-of-plane tractions $T_{z}$, and displacements $u_{z}$ are neglected, the accuracy of the in-plane monolayer stresses would, in principle, be affected in two ways. (i) Neglecting the out-of-plane components can have an indirect effect on the monolayer stresses via the errors introduced in the in-plane tractions. However, Fig. 2 shows that the effect of neglecting $u_{z}$ on the recovered in-plane tractions is remarkably small. Hence, the indirect effects on the monolayer stresses are also expected to be small. (ii) Neglecting the out-of-plane components can have a direct effect on the monolayer stresses. In the limit of classical thin plate theory, $T_{z}$ will induce bending moments in the monolayer, whose contribution to $\sigma_{x x}, \sigma_{y y}$, and $\sigma_{x y}$ scale as $z T_{z} l^{2} / h^{3}$, where $z$ has its origin at the mid-plane of the monolayer. The thickness average of this contribution is, however, identically zero. In connection with the distribution of three-dimensional tractions, Notbohm et al. ([29]; Fig. 6a) propose a model, according to which both $T_{z}$ and associated bending moments are largely confined to the monolayer boundary. Near the boundary, in the limit of classical plate theory the thickness averaged monolayer stresses are negligible. Away from the boundary, the monolayer stresses have even higher accuracy. For example, beyond a distance proportional to the height of the monolayer, according to St. Venant's principle, the bending moments and, therefore, the errors in monolayer stresses will be negligible (Note that this argument is not restricted to the classical thin plate theory). Taken together, the three-dimensional traction model of Notbohm et al. [29] suggests that monolayer stresses recovered by neglecting $T_{z}$ would have associated errors that are negligible.

Moreover, it has been suggested in several reports that the problem of intercellular stress recovery, given the traction field, is mathematically intractable $[19,28,39]$. It is true that there are boundary artifacts caused by optical edges which introduce slowly varying but unknown additive terms. Measurements from an island, which has no optical edge, suffer from no such artifact, however, and, as such, monolayer stresses can be determined uniquely. Further, direct comparison of stress distributions computed for an entire island compared with stress distributions computed for a sub-region of interest bounded by optical edges (assuming appropriate boundary conditions) show only modest differences after cropping (Figs. 5k, S3k, S5k). In summary, for isolated islands the problem of recovery of stress distributions is mathematically tractable; for monolayers extending outside the field-of-view, the stress distributions can be estimated accurately using MSM with suitable care.

\section{Boundary artifacts are confined largely to the boundary}

Ad hoc boundary conditions at the optical edge introduced systematic artifacts in the recovered distribution of monolayer stresses, especially near optical edges. Near the optical edge, these artifacts were heterogeneous and large (Fig. $5 \mathrm{~g}-\mathrm{i}$, region shaded in grey, and Fig. $5 \mathrm{j}-1$, blue points). However, away from the optical edge these artifacts were largely homogeneous and quite small (Fig. 5g-i, non-shaded region, and Fig. 5j-l, red points). These findings confirm and extend the analysis of Tambe et al. [22].

Subsequent to Tambe et al. [22], Hur et al. [28] analyzed a square monolayer region bounded on all four sides by optical edges. At the optical edges, instead of assuming symmetric boundary conditions (see Introduction, Boundary Conditions and [22]), Hur et al. [28] assumed stress-free boundary conditions, which mechanically uncouples the region of interest from the rest of the monolayer. To avoid unknown influences of cells outside the optical field-of-view, Hur et al. [28] then cropped their stress maps but much less so (by a factor of six) than did Tambe et al. [22]. Monolayer stresses computed with the cropping and boundary conditions of Hur et al. [28] had a poor correlation $r^{2}=0.58$ with artifact-free stresses computed from an entire island. By contrast, monolayer stresses obtained using the cropping and boundary conditions of Tambe et al. [22], were tightly correlated $r^{2}=0.98$ with stresses computed from the entire island. (Fig. S5k, red points, in File S1).

\section{Artifacts attributable to material properties are small}

On the one hand, a recent study [40] makes the striking observation that across a monolayer the cell stiffness is extremely homogeneous. On the other hand, in isolated cells we have previously established a direct proportionality between cytoskeletal stress and cytoskeletal stiffness [31-33], which we assumed here. As regards heterogeneity of elastic cellular properties within a monolayer, therefore, these extreme cases would seem to bound the plausible possibilities.

If monolayer material properties are homogeneous, then stress recovery using MSM is unaffected. If the properties are heterogeneous (Fig. 4b), recovered stresses at high stress magnitudes were slightly underestimated, and at low stress magnitudes were slightly overestimated (Fig. 4k, blue points). Therefore, although the recovered stress landscape would be slightly flattened, the ruggedness of the stress landscape remains a robust finding. 


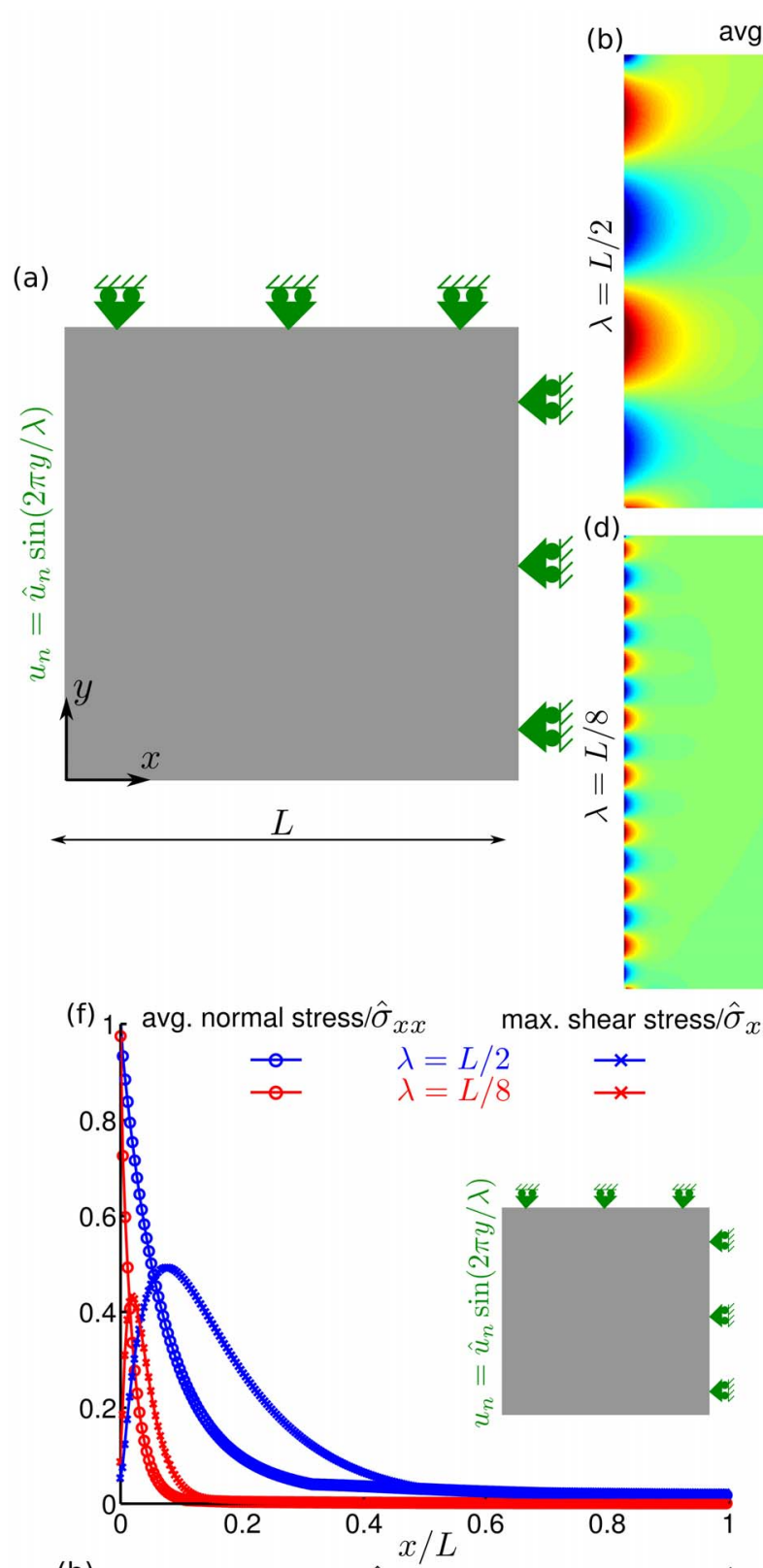

(h) 1 a avg. normal stress $/ \hat{\sigma}_{y y}$ max. shear stress $/ \hat{\sigma}_{y y}$

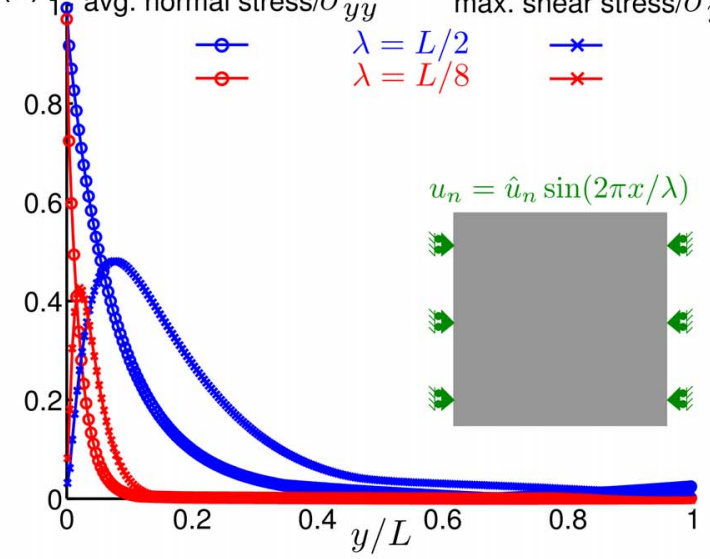

(c)

$\max$. shear stress $/ \hat{\sigma}_{x x}$

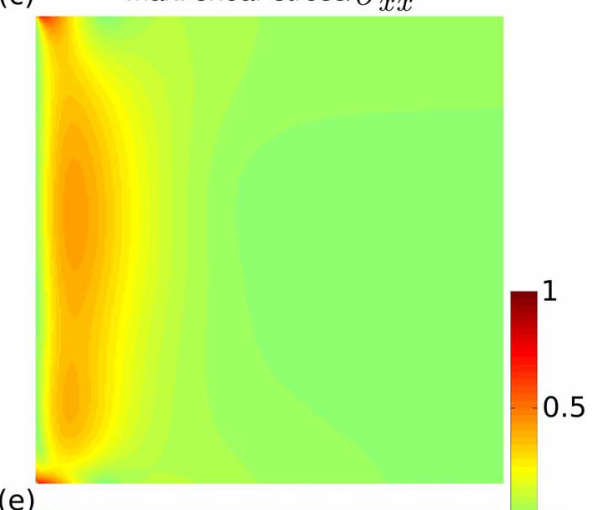

(e)

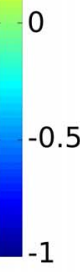

(i) 1 avg. normal stress $/ \hat{\sigma}_{x y} x / L$ max. shear stress $/ \hat{\sigma}_{x y}$
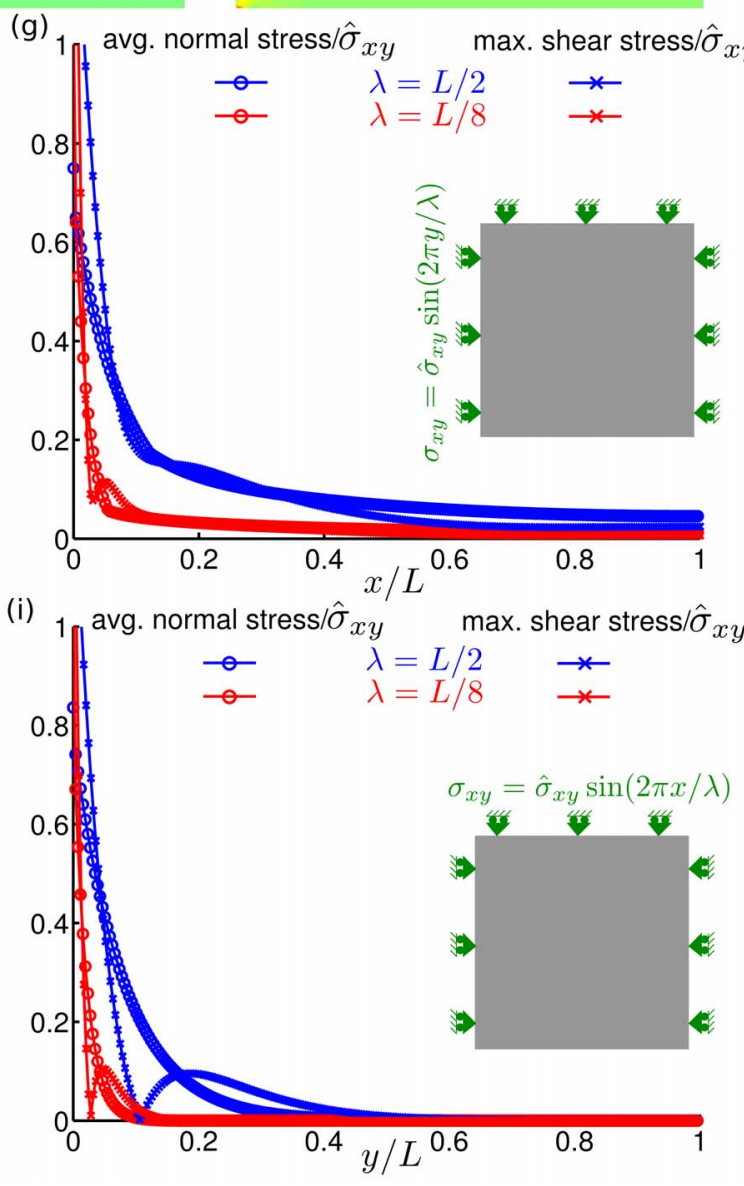

Figure 6. Propagation of boundary artifacts away from the optical edge. (a) A thin sheet subjected to sinusoidal perturbations in normal displacements $u_{n}(y)=\hat{u}_{n} \sin (2 \pi y / \lambda)$ at one edge, and $\mathrm{u}_{\mathrm{n}}=0$ at two other edges. (b) Map of average normal stress, and (c) map of maximum shear stress when $\lambda=\mathrm{L} / 2$. (d-e) The stress maps when $\lambda=\mathrm{L} / 8$. (f) Decay of dominant Fourier mode in the stresses induced by the boundary conditions 
shown in the inset. Blue curves correspond to $\lambda=\mathrm{L} / 2$, and red curves correspond to $\lambda=\mathrm{L} / 8$. The curves marked with circle represent the induced average normal stress, and the curves marked with cross represent the induced maximum shear stress. (g-i) Decay curves of the stresses induced by boundary conditions shown in the inset. At all the boundaries along appropriate axis the natural boundary conditions, i.e. boundary stress $=0$ are not mentioned but they are implied. The stresses in $(b-f)$ are normalized with the amplitude of induced normal stress $\hat{\sigma}_{x x}$ at the perturbed edge, the stresses in $(g, i)$ are normalized with amplitude of imposed shear stress $\hat{\sigma}_{x y}$, and the stresses in $(\mathrm{h})$ are normalized with the amplitude of induced normal stress $\hat{\sigma}_{y y}$ at the perturbed edge.

doi:10.1371/journal.pone.0055172.g006

The assumption of incompressibility caused no such artifacts, however (Fig. 4j-l, red points). Moreover, the prediction of local cell orientation by local maximum principal stress orientation was robust (Supporting Information S3; Fig. S1; in File S1). As such, recovered stresses depended upon assigned material properties, as expected, but the magnitude of these effects was unexpectedly small.

Although movement of the epithelial sheet has been shown to respond to the viscosity of the substrate [41], here we take the substrate to be simply and linearly elastic, as in traditional polyacrylamide and PDMS. As for the cell monolayer itself, most mathematical models of development assume that the dominant mechanical stress in cells comprising the monolayer is viscous on the slow time scales relevant to migration [42]. Recent experimental data demonstrate, to the contrary, that the dominant mechanical stress in cells comprising the monolayer is very nearly elastic [23]. Moreover, since the monolayer stress recovery rests not on dynamic but on instantaneous balance of the substrate tractions, neglecting viscosity of the monolayer has no associated artifact.

\section{The observation of plithotaxis is robust}

According to the principle of plithotaxis, the local orientation of cell migration and the local orientation of maximum principal stress are strongly associated [22]. Might the artifacts addressed here impact this finding? The maximum principal orientation was found to be largely insensitive to the artifacts addressed here (Figs. 5j, S3j, and S5j), thus leaving the finding of plithotaxis unaltered.

The association between the stress and the motion is related to the local stress anisotropy; the higher is the anisotropy, the tighter is the association [22]. Although the finding of plithotaxis was insensitive to the artifacts addressed here, the anisotropy of the stress (i.e. the maximum shear stress) was, however, affected by the boundary artifacts (Fig. 5l, after data cropping, $r^{2}=0.66$ ). Nonetheless, in the regions where anisotropy was highest and lowest, the degree of anisotropy was largely preserved (data not shown). Hence, the observation that higher stress anisotropy lead to tighter mechanical guidance is also robust.

Based upon this evidence and these arguments, we agree that out-of-plane tractions may be present and are of some interest.

\section{References}

1. Alberts B (2009) Redefining Cancer Research. Science 325: 1319.

2. Butcher DT, Alliston T, Weaver VM (2009) A tense situation: forcing tumour progression. Nat Rev Cancer 9: 108-122.

3. Discher D, Dong C, Fredberg.JJ, Guilak F, Ingber D, et al. (2009) Biomechanics: cell research and applications for the next decade. Ann Biomed Eng 37: 847859

4. Discher DE, Janmey P, Wang YL (2005) Tissue cells feel and respond to the stiffness of their substrate. Science 310: 1139-1143.

5. Eisenhoffer GT, Loftus PD, Yoshigi M, Otsuna H, Chien C-B, et al. (2012) Crowding induces live cell extrusion to maintain homeostatic cell numbers in epithelia. Nature advance online publication.

6. Bosveld F, Bonnet I, Guirao B, Tlili S, Wang Z, et al. (2012) Mechanical Control of Morphogenesis by Fat/Dachsous/Four-Jointed Planar Cell Polarity Pathway. Science 336: 724-727.

7. Engler AJ, Humbert PO, Wehrle-Haller B, Weaver VM (2009) Multiscale modeling of form and function. Science 324: 208-212.
However, out-of-plane tractions are ordinarily uncoupled from, and therefore have no appreciable effects upon, recovered in-plane stresses.

\section{Conclusions}

For the cellular monolayer in vitro, MSM maps thicknessaveraged in-plane components of the complete two-dimensional stress tensor [22]. Although MSM ignores out-of-plane stress components, it recovers in-plane stress components with negligible error and without the requirements for confocal microscopy and computational resources required for three-dimensional reconstructions $[28,29]$. MSM is not subject to the additional errors that might be attributable to experimental imprecision in resolving $z$ displacements, moreover, or to computational error in threedimensional finite element analysis. Stress recovery using MSM is simpler than three dimensional approaches and is insensitive to those artifacts that have been identified.

\section{Supporting Information}

File S1 Supporting Information S1-S8. Details of experimental and mathematical approach and accuracy assessment for two other monolayer geometries of interest. The supporting information contains several topics which includes, protocol for mapping stresses ( $\mathrm{S} 1)$, Fourier representation of three dimensional Boussinesq solution (S2), data for alignment between maximum principal orientation and cell orientation (S3), accuracy assessment for two other monolayer systems of interest (S4), effect of adjacent free edges on rate of decay of boundary artifacts (S5), procedure for mapping decay of boundary artifacts (S6), effect of substrate thickness on substrate tractions (S7), and enlarged images of selected results (S8).

(PDF)

\section{Author Contributions}

3D traction analysis: JPB. Discussed and interpreted the results and commented on the manuscript: DTT UC XT CYP JHK EM JPB JJF. Conceived and designed the experiments: DTT JPB JJF. Performed the experiments: DTT UC. Analyzed the data: DTT JPB. Wrote the paper: DTT JPB JJF.

8. Friedl P, Gilmour D (2009) Collective cell migration in morphogenesis, regeneration and cancer. Nat Rev Mol Cell Biol 10: 445-457.

9. Kumar S, Weaver VM (2009) Mechanics, malignancy, and metastasis: the force journey of a tumor cell. Cancer Metastasis Rev 28: 113-127.

10. Levental KR, Yu H, Kass L, Lakins JN, Egeblad M, et al. (2009) Matrix crosslinking forces tumor progression by enhancing integrin signaling. Cell 139: 891-906.

11. Marinari E, Mehonic A, Curran S, Gale J, Duke T, et al. (2012) Live-cell delamination counterbalances epithelial growth to limit tissue overcrowding. Nature advance online publication.

12. Paszek MJ, Boettiger D, Weaver VM, Hammer DA (2009) Integrin clustering is driven by mechanical resistance from the glycocalyx and the substrate. PLoS Comput Biol 5: e1000604.

13. Paszek MJ, Weaver VM (2004) The tension mounts: mechanics meet morphogenesis and malignancy. J Mammary Gland Biol Neoplasia 9: 325-342. 
14. Woolner S, Papalopulu N (2012) Spindle Position in Symmetric Cell Divisions during Epiboly Is Controlled by Opposing and Dynamic Apicobasal Forces. Developmental cell 22: 775-787.

15. Roh-Johnson M, Shemer G, Higgins CD, McClellan JH, Werts AD, et al. (2012) Triggering a Cell Shape Change by Exploiting Preexisting Actomyosin Contractions. Science 335: 1232-1235.

16. Bagorda A, Parent CA (2008) Eukaryotic chemotaxis at a glance. Journal of Cell Science 121: 2621-2624.

17. Lo C-M, Wang H-B, Dembo M, Wang Y-1 (2000) Cell Movement Is Guided by the Rigidity of the Substrate. Biophys J 79: 144-152.

18. McCarthy JB, Palm SL, Furcht LT (1983) Migration by haptotaxis of a Schwann cell tumor line to the basement membrane glycoprotein laminin. J Cell Biol 97: $772-777$.

19. Liu Z, Tan JL, Cohen DM, Yang MT, Sniadecki NJ, et al. (2010) Mechanical tugging force regulates the size of cell-cell junctions. Proc Natl Acad Sci U S A 107: $9944-9949$

20. Maruthamuthu V, Sabass B, Schwarz US, Gardel ML (2011) Cell-ECM traction force modulates endogenous tension at cell-cell contacts. Proc Natl Acad Sci U S A 108: 4708-4713.

21. Gov N (2011) Cell mechanics: Moving under peer pressure. Nat Mater 10: 412 414.

22. Tambe DT, Hardin CG, Angelini TE, Rajendran K, Park CY, et al. (2011) Collective cell guidance by cooperative intercellular forces. Nat Mater 10: 469475.

23. Serra-Picamal X, Conte V, Vincent R, Anon E, Tambe DT, et al. (2012) Mechanical waves during tissue expansion. Nat Phys 8: 628-634.

24. Thery M (2012) Cell mechanics: Wave of migration. Nat Phys 8: 583-584.

25. Trepat X, Fredberg JJ (2011) Plithotaxis and emergent dynamics in collective cellular migration. Trends Cell Biol 21: 638-646.

26. du Roure O, Saez A, Buguin A, Austin RH, Chavrier P, et al. (2005) Force mapping in epithelial cell migration. Proc Natl Acad Sci U S A 102: 2390-2395.

27. Trepat X, Wasserman MR, Angelini TE, Millet E, Weitz DA, et al. (2009) Physical forces during collective cell migration. Nat Phys 5: 426-430.

28. Hur SS, del Alamo JC, Park JS, Li YS, Nguyen HA, et al. (2012) Roles of cell confluency and fluid shear in 3-dimensional intracellular forces in endothelial cells. Proc Natl Acad Sci U S A 109: 11110-11115.
29. Notbohm J, Kim JH, Asthagiri AR, Ravichandran G (2012) Three-Dimensional Analysis of the Effect of Epidermal Growth Factor on Cell-Gell Adhesion in Epithelial Cell Clusters. Biophys J 102: 1323-1330.

30. Fung YC (1993) Biomechanics: Mechanical Properties of Living Tissues: Springer.

31. Wang N, Naruse K, Stamenovic D, Fredberg JJ, Mijailovich SM, et al. (2001) Mechanical behavior in living cells consistent with the tensegrity model. Proc Natl Acad Sci U S A 98: 7765-7770.

32. Solon J, Levental I, Sengupta K, Georges PC, Janmey PA (2007) Fibroblast Adaptation and Stiffness Matching to Soft Elastic Substrates. Biophys J 93: 4453-4461.

33. Park CY, Tambe D, Alencar AM, Trepat X, Zhou EH, et al. (2010) Mapping the cytoskeletal prestress. American Journal of Physiology - Cell Physiology 298: C1245-C1252.

34. Del Alamo JC, Meili R, Alonso-Latorre B, Rodriguez-Rodriguez J, Aliseda A, et al. (2007) Spatio-temporal analysis of eukaryotic cell motility by improved force cytometry. Proc Natl Acad Sci U S A 104: 13343-13348.

35. Lin Y-C, Tambe DT, Park CY, Wasserman MR, Trepat X, et al. (2010) Mechanosensing of substrate thickness. Physical Review E 82: 041918.

36. Butler JP, Tolic-Norrelykke IM, Fabry B, Fredberg JJ (2002) Traction fields, moments, and strain energy that cells exert on their surroundings. Am J Physiol Cell Physiol 282: C595-605.

37. Engler A, Bacakova L, Newman C, Hategan A, Griffin M, et al. (2004) Substrate Compliance versus Ligand Density in Cell on Gel Responses. Biophys J 86: 617628.

38. Landau LD, Pitaevskii LP, Lifshitz EM, Kosevich AM (1986) Theory of Elasticity: Butterworth-Heinemann.

39. Ting LH, Jahn JR, Jung JI, Shuman BR, Feghhi S, et al. (2012) Flow mechanotransduction regulates traction forces, intercellular forces, and adherens junctions. American Journal of Physiology - Heart and Circulatory Physiology 302: H2220-H2229.

40. Harris AR, Peter L, Bellis J, Baum B, Kabla AJ, et al. (2012) Characterizing the mechanics of cultured cell monolayers. Proceedings of the National Academy of Sciences.

41. Murrell M, Kamm R, Matsudaira P (2011) Substrate Viscosity Enhances Correlation in Epithelial Sheet Movement. Biophysical journal 101: 297-306.

42. Chen X, Brodland GW (2008) Multi-scale finite element modeling allows the mechanics of amphibian neurulation to be elucidated. Phys Biol 5: 015003. 